Dynamic characteri sti c anal ysi s of nonr esonance- type ul trasoni $c$ act uat or usi ng el ectroni c ci rcui $t$ si mul at or

\begin{tabular}{|l|l|}
\hline 著者 & SAKAl Shi nj i , AOYAG Nanabu \\
\hline $\begin{array}{l}\text { j our nal or } \\
\text { publ i cat i on t i t l e }\end{array}$ & Japanese J our nal of Appl i ed Physi cs \\
\hline vol une & 54 \\
\hline number & $7 S 1$ \\
\hline year & $2015-06-22$ \\
\hline URL & ht t p: //hdl . handl e. net /10258/00008881 \\
\hline
\end{tabular}


Dynamic characteri sti c anal ysi s of nonr esonance- type ul trasoni $c$ act uat or usi ng el ectroni c ci rcui $t$ si mul at or

\begin{tabular}{|l|l|}
\hline 著者 & SAKAl Shi nj i, AOYAG Nanabu \\
\hline $\begin{array}{l}\text { j our nal or } \\
\text { publ i cat i on ti tl e }\end{array}$ & Japanese J ournal of Appl i ed Physi cs \\
\hline vol une & 54 \\
\hline number & 7 S1 \\
\hline year & 2015-06-22 \\
\hline URL & ht t p: //hdl . handl e. net /10258/00008881 \\
\hline
\end{tabular}




\title{
Dynamic characteristic analysis of nonresonance-type ultrasonic actuator using electronic circuit simulator
}

\author{
Shinji Sakai and Manabu Aoyagi*
}

Graduate School of Engineering, Muroran Institute of Technology, Muroran, Hokkaido 050-8585, Japan

\begin{abstract}
The electromechanical equivalent circuit of an ultrasonic actuator is useful for gaining intuitive understanding or for design. However, the equivalent circuit had not been simulated directly on commercial computer software such as an electronic circuit simulator (ECS), because the nonlinear elements that express friction or contact separation are rarely expressed on the equivalent circuit. In this paper, the equivalent circuit model of a nonresonance-type ultrasonic actuator, which is created on the basis of the equation of motion and can be simulated on a commercial ECS, is proposed, and some simulated results obtained using a commercial ECS are reported.
\end{abstract}

\section{Introduction}

An ultrasonic actuator generates driving force by frictional force of ultrasonic vibration. The ultrasonic actuator has a nonlinear element such as contact separation and slip stick. Many analyses of the driving force have been reported. Those analyses employed the dynamic model, ${ }^{1-3)}$ equivalent circuit, ${ }^{4-7)}$ or finite element method (FEM) ${ }^{8)}$ The FEM simulation clarifies the mechanical characteristics of the ultrasonic actuator. However, the FEM is not convenient for design because of long calculation time and complex modeling. In the case of the simulation with the dynamic model, it is necessary to write the numerical analysis program to solve the equation of motion. Similarly, a writing program is required for the simulation using the equivalent circuit.

An ultrasonic actuator is a transducer that converts electrical energy into mechanical energy. Electrical and mechanical systems can find analogies with equivalent circuits. ${ }^{9,10)}$ Therefore, the electromechanical equivalent circuit is useful for gaining intuitive understanding or for design. The application of the equivalent circuit to piezoelectric devices has been reported, for example, for the force estimation of a piezoelectric actuator by monitoring driving current, ${ }^{11)}$ the control of piezoelectric actuator, ${ }^{12)}$ characteristic analyses of a piezoelectric gyroscope $^{13,14)}$ or a piezoelectric transformer, ${ }^{15,16)}$ and analyses of spurious mode control of 
an ultrasonic motor using electrical elements connected at electrical output ports. ${ }^{17)}$

In this study, we attempted to simulate the dynamic characteristic of the ultrasonic actuator by using an electronic circuit simulator (ECS). The equivalent circuit on the ECS can include electronic circuit elements for the reproduction of a nonlinear phenomenon of the ultrasonic actuator. If a nonlinear element could be defined on an ECS, the dynamics of an ultrasonic actuator might be analyzed directly and easily on the ECS. Although such a situation was studied, a practical equivalent circuit was not obtained. ${ }^{18)}$ In this paper, the equivalent circuit model of a nonresonance-type ultrasonic actuator, which can be directly simulated on a commercial ECS, is proposed. Some performances of the ultrasonic actuator simulated by the ECS are reported.

\section{Dynamic model of ultrasonic actuator}

Figure 1 shows the basic construction of an ultrasonic actuator composed of piezoelectric actuators as the stator and the rotor. The piezoelectric actuators and a base are arranged to form a right triangle, as shown in Fig. 1. A preload is applied using a spring between the piezoelectric actuator and rotor. The rotor rotates owing to friction when the stator is in contact with the rotor. The top of the stator undergoes nonlinear motion by contact and separation with the rotor. Dynamic models of normal and tangential components of the ultrasonic actuator in the contact surface are shown in Figs. 2 and 3, respectively. In both figures, $K$ is the spring constant $(\mathrm{N} / \mathrm{m}), D$ denotes the damping coefficient $(\mathrm{N} \cdot \mathrm{s} / \mathrm{m})$, and $M$ is the mass $(\mathrm{kg})$. The mass of the piezoelectric actuator, which is divided equally, can be placed at the two ends in the dynamic model under the condition that the piezoelectric actuator operates at a lower frequency than its own resonance frequency. ${ }^{19)}$ When the stator is in contact with the transfer object, the normal force is applied at the contact surface. Meanwhile, the friction force occurs in proportion to the normal force. In the dynamic model, it is assumed that the dynamic friction coefficient is equal to the static one for the simplification of the equivalent circuit.

The applied force in the normal direction of the piezoelectric actuator is set as $f_{m 1}=$ $F_{m 1} e^{\mathrm{j} \omega \mathrm{t}}$. The equation of motion in the normal direction is given by

$$
\begin{aligned}
& F_{c}-\left\{j \omega\left(M_{p}+\frac{M_{0}}{2}\right)+D_{p}+\frac{K_{p}}{j \omega}\right\} \dot{u}_{s 1} \\
= & f_{m 1}-\left(D_{m 1}+\frac{K_{m 1}}{j \omega}\right)\left(\dot{u}_{s 2}-\dot{u}_{s 1}\right)
\end{aligned}
$$




$$
=\left\{\begin{array}{c}
\left(j \omega \frac{M_{0}}{2}+D_{N}+\frac{K_{N}}{j \omega}\right) \dot{u}_{r} \quad\left(u_{s 2}=u_{r}\right) \\
j \omega \frac{M_{0}}{2} \dot{u}_{s 2} \quad\left(u_{s 2}<u_{r}\right),
\end{array}\right.
$$

where $F_{c}$ is the preload force; $\dot{u}_{s 1}$ and $\dot{u}_{s 2}$, the velocity of the two ends of the piezoelectric actuator; and $\dot{u}_{r}$, the velocity of the rotor. The applied force in the normal direction on the rotor is given by

$$
f_{N}=\left\{\begin{array}{c}
\left(D_{N}+\frac{K_{N}}{j \omega}\right) \dot{u}_{r} \quad\left(u_{s 2}=u_{r}\right) \\
0 \quad\left(u_{s 2}<u_{r}\right) .
\end{array}\right.
$$

The applied force in the tangential direction of the piezoelectric actuator is set as $f_{m 2}=$ $F_{m 2} e^{j \omega t}$. The equation of motion in the tangential direction is given by

$$
\begin{aligned}
& f_{m 2}-\left(j \omega \frac{M_{0}}{2}+D_{m 2}+\frac{K_{m 2}}{j \omega}\right) \dot{u}_{s t} \\
= & \left(j \omega M_{T}+D_{\text {loss }}\right) v_{T} \\
= & f_{T} \\
= & \left\{\begin{array}{cc}
\mu f_{N} \operatorname{sgn}\left(\dot{u}_{s T}-v_{r}\right) \quad(\text { slip }) \\
K_{T}\left(v_{r}-v_{T}\right) & (\text { stick }),
\end{array}\right.
\end{aligned}
$$

where $\dot{u}_{s T}$ is the velocity of the stator; $\dot{v}_{T}$, the velocity of the rotor; $D_{\text {loss }}$, the damping coefficient of the rotor; and $v_{r}$, the velocity in the contact surface of the rotor. The loss of the rotor is expressed as $D_{\text {loss }} v_{T}$.

\section{Mechanodynamically equivalent circuit}

\subsection{Design of mechanodynamically equivalent circuit}

The mechanodynamically equivalent circuit of the nonresonance-type ultrasonic actuator developed from Eqs. (1)-(3) is shown in Fig. 4. The equivalent circuit is drawn using the symbols of electric and electronic circuit elements of the commercial ECS (Myway Plus, PSIM). The voltage and electric current correspond to the force and velocity, respectively. In the normal component, the switch and diode are contact-separation factors. The switch $S_{C}$ is turned off while the stator is in contact with the rotor by the output of comparator Comp., which compares the position of the rotor with stator. Those factors enable the analysis of the nonlinear normal contact force. 
In the tangential component, the voltage-controlled voltage sources (VCVSs) and diodes are friction factors. The VCVS outputs a voltage that is proportional to the voltage of the normal component. To understand the operation of the VCVS, the simple limit circuit using the VCVSs is shown in Fig. 5(a), and voltage waveforms of input $V_{\text {in }}$ and output $V_{\text {out }}$ are shown in Fig. 5(b). The VCVSs with control voltage of $100 \mathrm{~V}$ and gain of 0.5 output $50 \mathrm{~V}$, so that the absolute value of $V_{\text {out }}$ is limited to $50 \mathrm{~V}$ or less. In Fig. 4, the control voltage of VCVSs is the output voltage $V_{C}$ of the normal component circuit, and represents the normal contact force. Therefore, if the gain value of a VCVS corresponds to a friction coefficient $\mu$, the limit circuit in the friction factor can equivalently express the slip and stick of friction. The nonlinear tangential friction force can be simulated using the VCVSs.

The equation of the circuit can correspond to the equation of motion. The correspondence between the parameter of the equivalent circuit and that of the dynamic model is shown in Table I. The resistance is the damping coefficient; the capacitance, the compliance; the reactance, the mass; $f_{m 1}$ and $f_{m 2}$, forces of the piezoelectric actuator in the normal and tangential directions, respectively; and $D_{\text {loss }}$, the damping coefficient of a transfer object.

\subsection{Comparison of simulation results}

The ultrasonic actuator was simulated by both the developed equivalent circuit and a numerical analysis program ${ }^{2)}$ for the dynamic model. The Runge-Kutta method was employed to solve the dynamic model of motion. The equivalent circuit simulation was carried out on the commercial ECS (Myway Plus, PSIM), which is effective for the analysis of the power electronics circuit. Ideal devices are provided on PSIM. Sinusoidal forces were applied to stator sections in the normal and tangential components, respectively. The phase difference between the tangential force $V_{2}$ and the normal force $V_{1}$ was $90^{\circ}$. Each parameter was set to actual values of the tested ultrasonic actuator. A time step of simulation $\Delta \mathrm{t}$ of $5 \mathrm{~ns}$ was chosen. Even when $\Delta \mathrm{t}$ was smaller than $5 \mathrm{~ns}$, it was confirmed that simulated results were the same.

The velocity of a transfer object and the instantaneous value of driving force in the steady state were simulated using both the equivalent circuit and the dynamic model, as shown in Figs. 6 and 7, respectively. Almost the same simulation results were obtained by the two simulation methods. Hence, the developed equivalent circuit can express the dynamic model of the ultrasonic actuator, and it was able to be analyzed directly on PSIM. 


\section{Electromechanical equivalent circuit}

\subsection{Design of electromechanical equivalent circuit}

It can be considered that the ultrasonic actuator is the transformer that converts electrical energy into mechanical energy. The electromechanical equivalent circuit ${ }^{19)}$ of the piezoelectric actuator is shown in Fig. 8(a). The input voltage is transformed into the force that is determined by the electromechanical coupling coefficient of the piezoelectric actuator. A force that is determined by the parameters of the piezoelectric actuator is applied at the two ends of the piezoelectric actuator. Considering the operating direction of the piezoelectric actuator, the equivalent circuit of the actuator can be expressed as in Fig. 8(b). The applied force is determined using trigonometric functions. The electromechanical equivalent circuit is designed using the circuits in Figs. 4 and 8(b). The ultrasonic actuator shown in Fig. 1 is described by the electrical equivalent circuit in Fig. 9. The phase difference between $V_{\text {in1 }}$ and $V_{\text {in2 }}$ is $90^{\circ}$. The ultrasonic actuator can be simulated by the electromechanical equivalent circuit in the ECS.

\subsection{Simulation results of electromechanical equivalent circuit in ECS}

The ultrasonic actuator using two multilayer piezoelectric actuators (MPAs) ${ }^{20-22)}$ was simulated using the circuit in the ECS shown in Fig. 9. The MPAs employed were AE0505D08 from NEC-Tokin Co. The parameter was set to the actual values of the tested ultrasonic actuator shown in Fig. 10. The values of the parameters used in the simulation are listed in Table II. Force factor, $A$, and stiffness of MPA, $K_{m}$, were set to the values on the datasheet provided by the manufacturer. The damping coefficient of MPA was determined from the force factor, the mass of MPA, $M_{0}$, the stiffness, $K_{m}$, the capacitance, $C_{d}$, and the measured value of the impedance of MPA at the driving frequency using the impedance analyzer. The simulated result is shown in Fig. 11. The input power is calculated using the driving voltage and electric current. The output power is calculated using the driving force and rotor velocity. Figure 11(a) shows the rise time of the rotor velocity. With a lower preload, the rise time is long and the velocity is high in the steady state. With a higher preload, the rise time becomes shorter and the velocity becomes lower in the steady state.

The preload control, which changes the preload continually or progressively in starting the ultrasonic actuator, can reduce the rise time of velocity while maintaining high velocity in the steady state. The preload was controlled depending on the velocity of the rotor as

$$
F_{c}=-245.48 v_{r}^{2}-50 v_{r}+50
$$


Equation (4) was determined from the envelope of the velocity and force characteristic curves shown in Fig. 11(b). The initial and steady preloads were $50.0 \mathrm{~N}$ and $0.042 \mathrm{~N}$, respectively. Figure 11(c) shows the efficiency characteristics. Using the preload control improves the characteristics of the force and velocity and the efficiency.

Figure 12 shows the comparison of simulated and measured results of velocity plotted against driving force. The two sets of results are not in agreement. The treatment of friction or parameters in the simulation using the ECS will be further examined.

\subsection{Application to improvement of ultrasonic actuator}

Figure 11(c) shows that the efficiency of the ultrasonic actuator is rather low when parameters are set as actual values. The improvement of the characteristics is examined by simulation using the ECS. The loss of the piezoelectric actuator is $96 \%$ of the input power, which is the highest among circuit elements. The piezoelectric material is assumed to be soft. The internal mechanical resistance of the soft-type piezoelectric material is higher than that of a the hard-type material. It is considered that the loss of the piezoelectric actuator is reduced by employing the hard-type piezoelectric material. The simulation result in the case of internal mechanical resistance reduced to one-tenth is shown in Fig. 13. It can be estimated that the efficiency is improved. On the other hand, it is considered that the efficiency can be improved by increasing the frictional coefficient between the stator and the rotor. The simulation result in the case of the frictional coefficient increasing from 0.1 to 0.3 is shown in Fig. 14. The improvements of the characteristics are estimated as well. Figure 15 shows the power flow of the ultrasonic actuator in the case of the reduction of the internal resistance and the increase of the frictional coefficient. It is estimated that the loss of slip and output mechanical power are smaller than the loss of the piezoelectric actuator. To improve the characteristics of the ultrasonic actuator, it is important that the loss of the piezoelectric actuator is reduced. Such simulations are easily carried out using the ECS.

\section{Summary}

The electromechanical equivalent circuit of the ultrasonic actuator that can be evaluated using in a commercial ECS was developed. Simulation results obtained using the equivalent circuit and dynamic model were almost equal. Hence, the developed equivalent circuit for the analysis of the ultrasonic actuator was effective for direct simulation using the commercial ECS. Such a simulation method is very useful for designing and evaluating the ultrasonic actuator. 


\section{References}

1) N. W. Hagood IV and A. J. McFarland, IEEE Trans. Ultrason. Ferroelectr. Freq. Control 42, 210 (1995).

2) T. Maeno, Nihon Onkyo Gakkaishi 54, 305 (1998)[in Japanese].

3) S. Gutschmidt and P. Hagedorn, Proc. Int. Conf. Acoust., 2004, p.I405.

4) Y. Tomikawa, M. Yaginuma, S. Hirose, and T. Takano, Jpn. J. Appl. Phys. 31, 2398 (1991).

5) K. Nakamura, M. Kurosawa, and S. Ueha, IEEE Trans. Ultrason. Ferroelectr. Freq. Control 38, 188 (1991).

6) M. Aoyagi and Y. Tomikawa, Jpn. J. Appl. Phys. 34, 2752 (1995).

7) M. Aoyagi, Y. Nakano, and Y. Tomikawa, Jpn. J. Appl. Phys. 34, 5292 (1995).

8) T. Maeno, T. Tsukimoto, and A. Miyake, IEEE Trans. Ultrason. Ferroelectr. Freq. Control 39, 668 (1992).

9) T. Sashida and T. Kenjo, An Introduction to Ultrasonic Motors (Clarendon Press, Oxford, U.K., 1993) p. 89.

10) S. Ueha, Y. Tomikawa, M. Kurosawa, and N. Nakamura, Ultrasonic Motors, Theory and Applications (Clearendon Press, Oxford, U.K., 1993) Chap. 7.

11) R. Inukai, K. Furutani, S. K. Chee, T. Yano, and T. Higuchi, Proc. Symp. Electromagnetics and Dynamics, 45 (2012) [in Japanese].

12) T. Yano, K. Yakuwa, A. Yano, S. K. Chee, and T. Higuchi, U.S. Patent 8552620 (2013).

13) S. Kudo, M. Konno, and K. Nakamura, Jpn. J. Appl. Phys. 29, 108 (1990).

14) M. Chiba and N. Wakatsuki, Jpn. J. Appl. Phys. 39, 3069 (2000).

15) M. Sato, M. Ueda, and H. Hasegawa, Jpn. J. Appl. Phys. 31, 181 (1991).

16) K. Sakurai, K. Ohnishi, and Y. Tomikawa, Jpn. J. Appl. Phys. 38, 5592 (1999).

17) M. Aoyagi, H. Watanabe, Y. Tomikawa, and T. Takano, Jpn. J. Appl. Phys. 41, 3252 (2002).

18) M. Aoyagi, M. Seki, T. Takano, H. Tamura, and Y. Tomikawa, Proc. Ultrasonic Electronics, 2008, p.497.

19) Mason, W. P, Physical Acoustics, (Academic Press, New York, 1964) Vol. 1, p.233.

20) T. Takemura, M. Aoyagi, T. Takano, H. Tamura, and Y. Tomikawa, Jpn. J. Appl. Phys. 47, 4265 (2008).

21) R. Okeya, K. Akiba, M. Aoyagi, H. Tamura, and T. Takano, Abstr. 28th Annu. Conf. Robotics Society of Japan, 2010, p.291 [in Japanese]. 
22) R. Okeya M. Aoyagi, T. Takano, and H. Tamura, Sens. Actuators A: Phys. 200, 155 (2013). 


\section{Figure Captions:}

Fig. 1 Basic construction of ultrasonic actuator.

Fig. 2 Dynamic model of normal component of ultrasonic actuator.

Fig. 3 Dynamic model of tangential component of ultrasonic actuator.

Fig. 4 Mechanodynamically equivalent circuit of ultrasonic actuator.

Fig. 5 Operation result of friction limit circuit using VCVSs. (a) Limit circuit. (b) Voltage waveforms of input and output.

Fig. 6 Simulated results of velocity of transfer object.

Fig. 7 Simulated results of instantaneous value of driving force in steady state.

Fig. 8 Electromechanical equivalent circuit of piezoelectric actuator. (a) The simple circuit.

(b) When the direction of the piezoelectric actuator is considered.

Fig. 9 Electromechanical equivalent circuit of ultrasonic actuator.

Fig. 10 Construction of ultrasonic actuator.

Fig. 11 Simulation results of electromechanical equivalent circuit with actual values of the tested ultrasonic actuator. (a) Rise time of the velocity of the rotor. (b) Velocity and force characteristics. (c) Efficiency characteristics.

Fig. 12 Simulation results and experimental results.

Fig. 13 Efficiency characteristic with internal mechanical resistance reduced to one-tenth.

Fig. 14 Efficiency characteristics with increasing frictional coefficient between stator and rotor from 0.1 to 0.3 .

Fig. 15 Example of the power flow of the ultrasonic actuator which employs internal mechanical resistance reduced to one-tenth and frictional coefficient between the rotor and stator increasing to 0.3 . 
Table I. Parameters of equivalent circuit and dynamic model.

\begin{tabular}{|c|c|c|}
\hline Element & Dynamic model & Equivalent circuit \\
\hline Force & $f_{m 1}, f_{m 2}, F_{c}$ & $V_{1}, V_{2}, V_{p}$ \\
\hline \multirow{2}{*}{$\begin{array}{c}\text { Damping } \\
\text { Coefficient }\end{array}$} & $D_{m 1}, D_{m 2}, D_{p}$ & $R_{m 1}, R_{m 2}, R_{p}$ \\
\cline { 2 - 3 } Compliance & $D_{N}, D_{\text {loss }}$ & $R_{N}, R_{T}$ \\
\hline & $1 / K_{m 1}, 1 / K_{m 2}$ & $C_{m 1}, C_{m 2}$ \\
\cline { 2 - 3 } & $1 / K_{p}$ & $C_{p}$ \\
\cline { 2 - 3 } & $1 / K_{N}, 1 / K_{T}$ & $C_{N}, C_{T}$ \\
\hline \multirow{4}{*}{ Mass } & $M_{0} / 2$ & $L_{m 1 a}$ \\
\cline { 2 - 3 } & $M_{0} / 2+M_{p}$ & $L_{m 1 b}$ \\
\cline { 2 - 3 } & $M_{0} / 2, M_{T}$ & $L_{m 2}, L_{T}$ \\
\hline
\end{tabular}


Table II. Parameters used in the calculation.

\begin{tabular}{|c|c|c|}
\hline Symbol & Mean & Value \\
\hline$V_{\text {in } 1}$ & Applied voltage & $20 \mathrm{Vp}-\mathrm{p}$ \\
\hline$V_{\text {in } 2}$ & Applied voltage & $20 \mathrm{Vp}-\mathrm{p}$ \\
\hline$F_{c}$ & Preload & - \\
\hline$A$ & Force factor & $5.75 \mathrm{~N} / \mathrm{V}$ \\
\hline$C_{d}$ & Capacitance of MPA & $0.75 \mu \mathrm{F}$ \\
\hline$D_{m}$ & Damping coefficient of MPA & $150 \mathrm{~N} \cdot \mathrm{s} / \mathrm{m}$ \\
\hline$M_{0}$ & Mass of MPA & $8 \times 10^{-3} \mathrm{~kg}$ \\
\hline$K_{m}$ & Stiffness of MPA & $93 \times 10^{6} \mathrm{~N} / \mathrm{m}$ \\
\hline$D_{p}$ & Damping coefficient of preload element & $100 \mathrm{~N} \cdot \mathrm{s} / \mathrm{m}$ \\
\hline$M_{p}$ & Mass of preload element & $0.2 \mathrm{~kg}$ \\
\hline$K_{p}$ & Spring constant of preload element & $0.169 \mathrm{~N} / \mathrm{m}$ \\
\hline$K_{N}$ & Normal spring constant of contact surface & $1.23 \times 10^{9} \mathrm{~N} / \mathrm{m}$ \\
\hline$D_{N}$ & Normal damping coefficient of contact surface & $100 \mathrm{~N} \cdot \mathrm{s} / \mathrm{m}$ \\
\hline$M_{T}$ & Equivalent mass of rotor & $0.49 \mathrm{~kg}$ \\
\hline$K_{T}$ & Tangential spring constant of contact surface & $1.23 \times 10^{10} \mathrm{~N} / \mathrm{m}$ \\
\hline$D_{\text {loss }}$ & Lrictional coefficient & $1.0 \times 10^{-3} \mathrm{~N} \cdot \mathrm{s} / \mathrm{m}$ \\
\hline$\mu$ & Angle of MPA & 0.1 \\
\hline$\theta$ & Driving frequency & $\pi / 4 \mathrm{rad}]$ \\
\hline$f$ & Loss of rotor & $23.5 \mathrm{kHz}$ \\
\hline
\end{tabular}




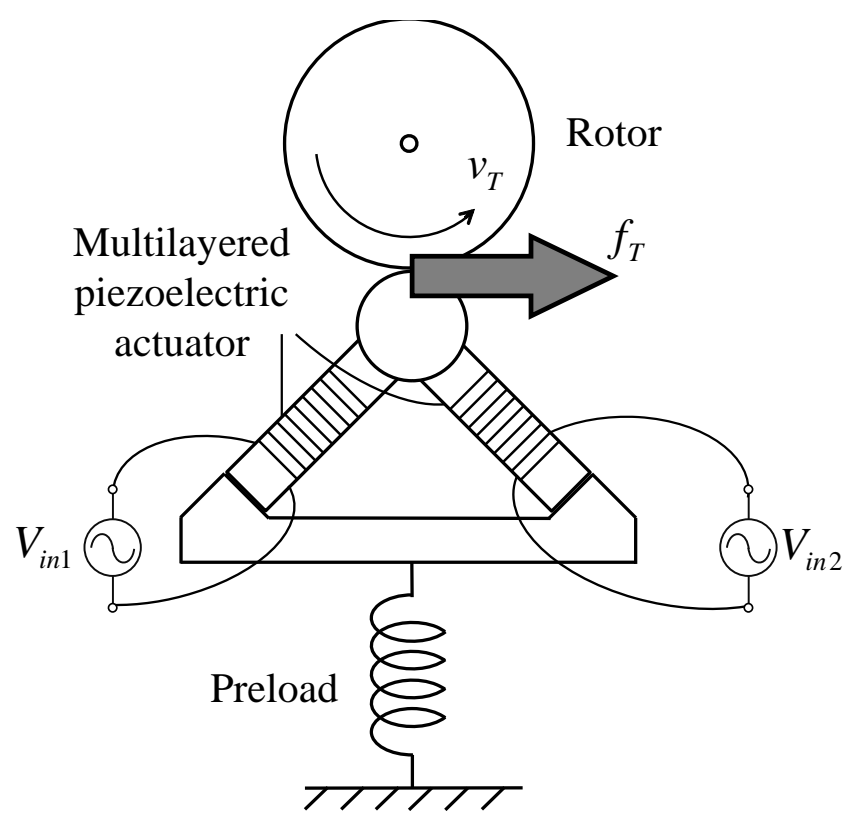

Fig. 1. Basic construction of ultrasonic actuator. 


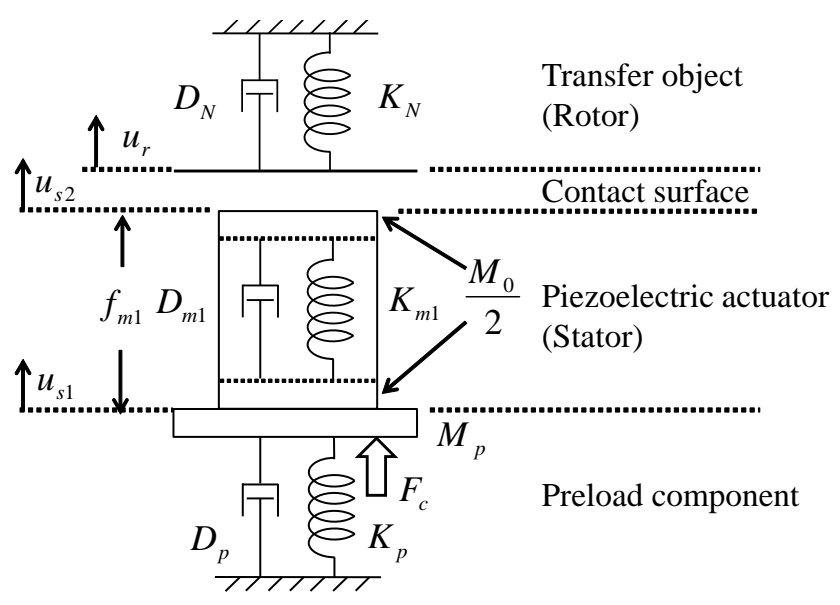

Fig. 2. Dynamic model of normal component of ultrasonic actuator. 


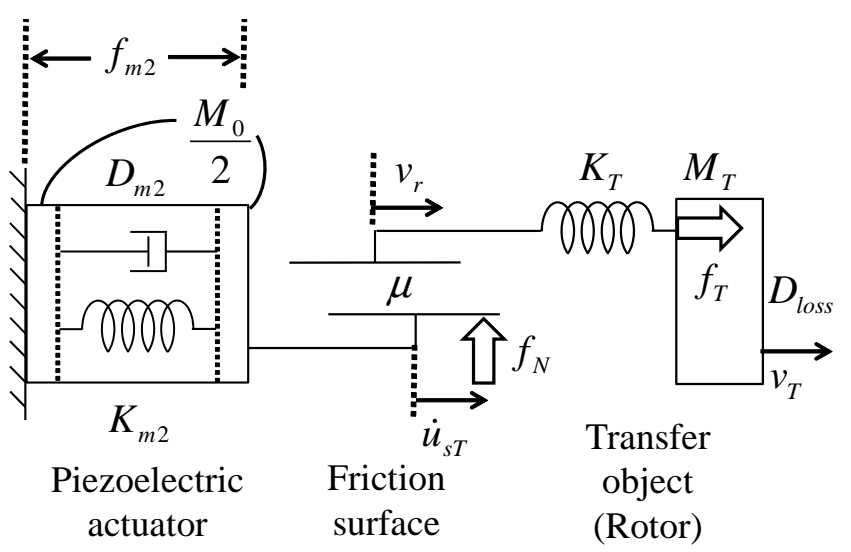

Fig. 3. Dynamic model of tangential component of ultrasonic actuator. 


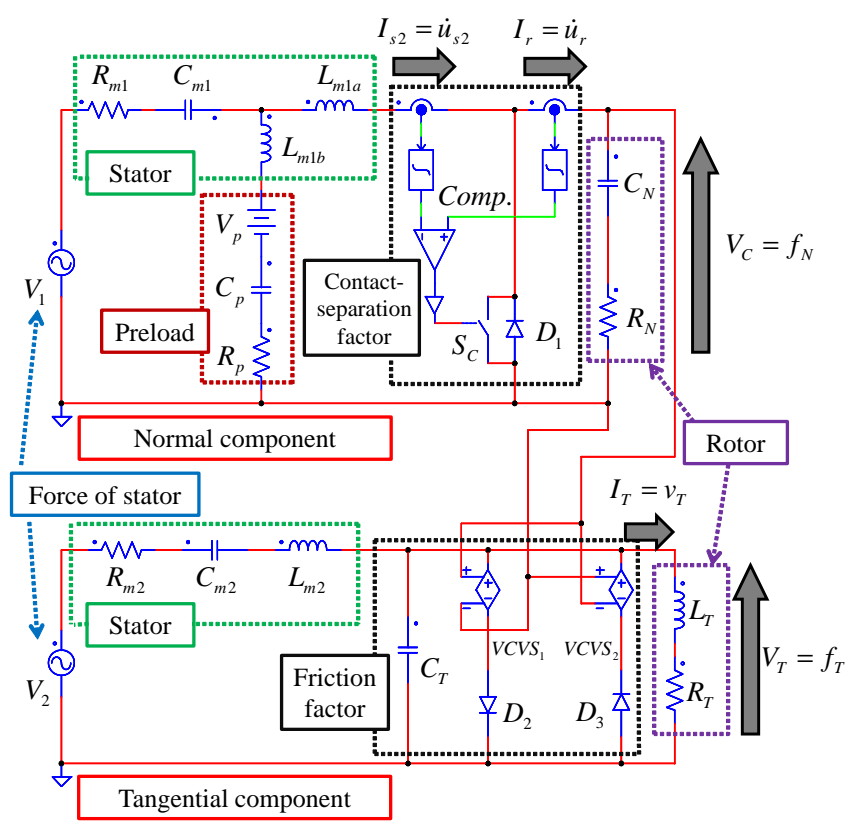

Fig. 4. Mechanodynamically equivalent circuit of ultrasonic actuator. 


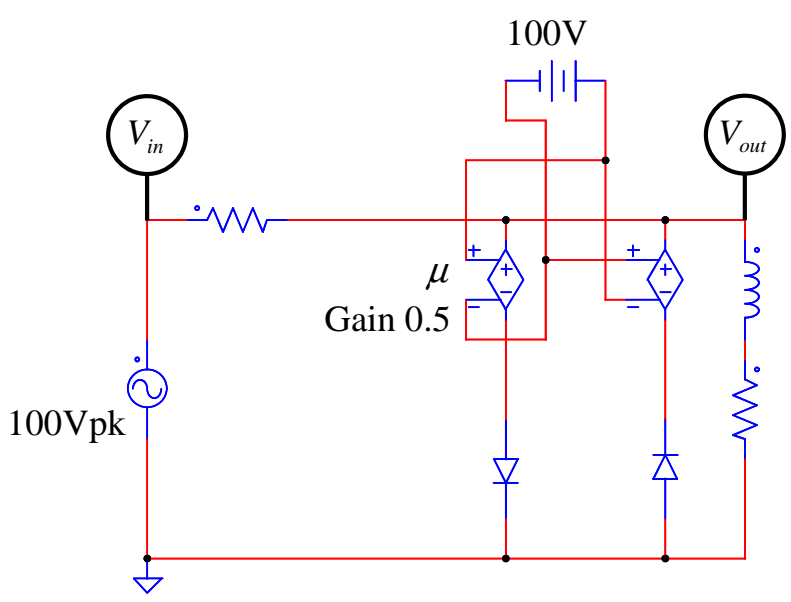

(a)

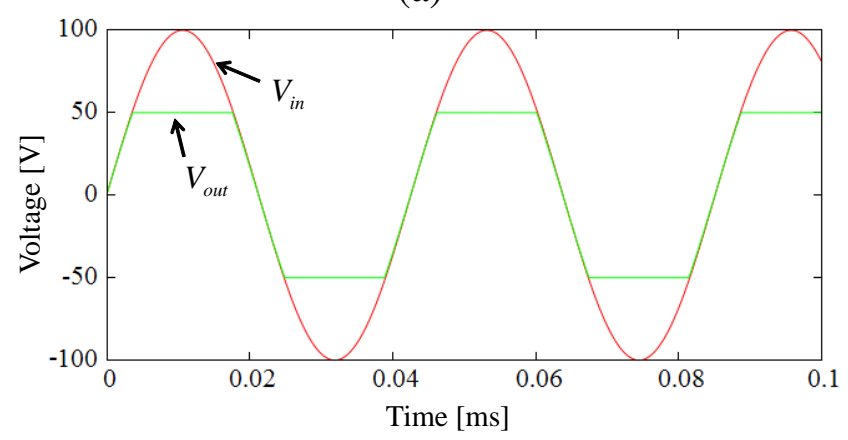

(b)

Fig. 5. Operation result of friction limit circuit using VCVSs. (a) Limit circuit. (b) Voltage waveforms of input and output. 


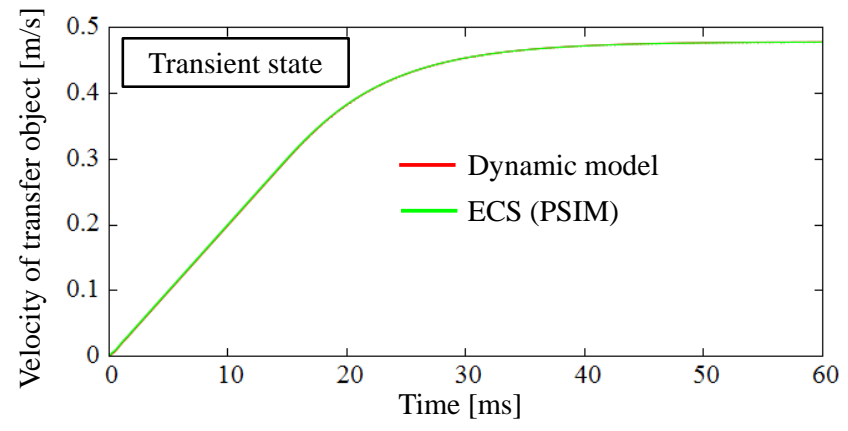

Fig. 6. Simulated results of velocity of transfer object. 


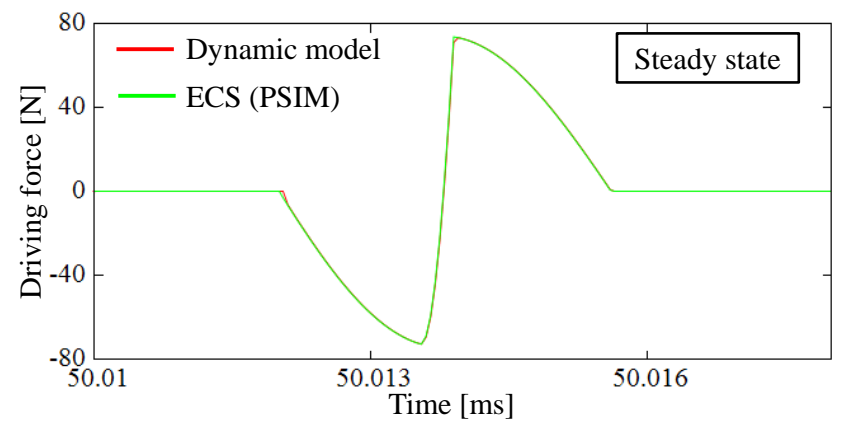

Fig. 7. Simulated results of instantaneous value of driving force in steady state. 


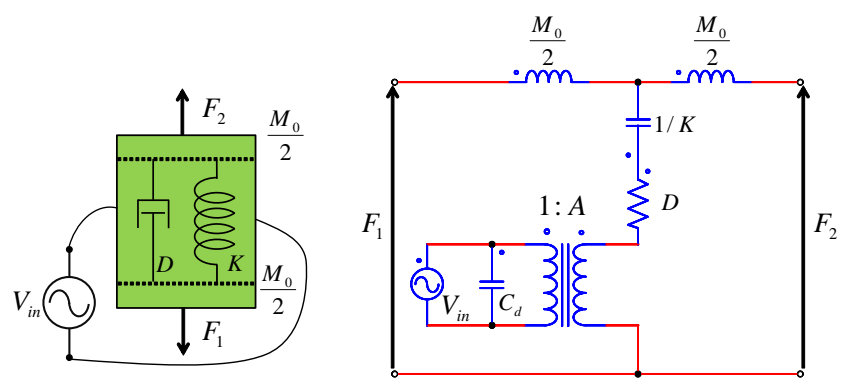

(a)
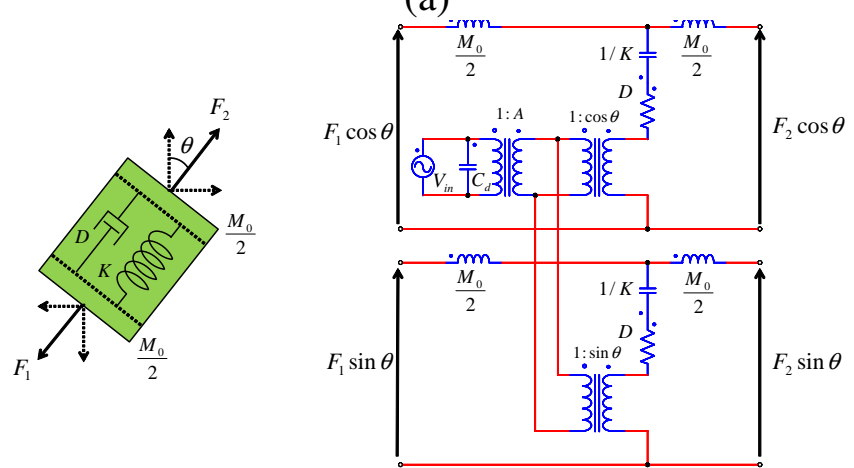

(b)

Fig. 8. Electromechanical equivalent circuit of piezoelectric actuator. (a) The simple circuit. (b) When the direction of the piezoelectric actuator is considered. 


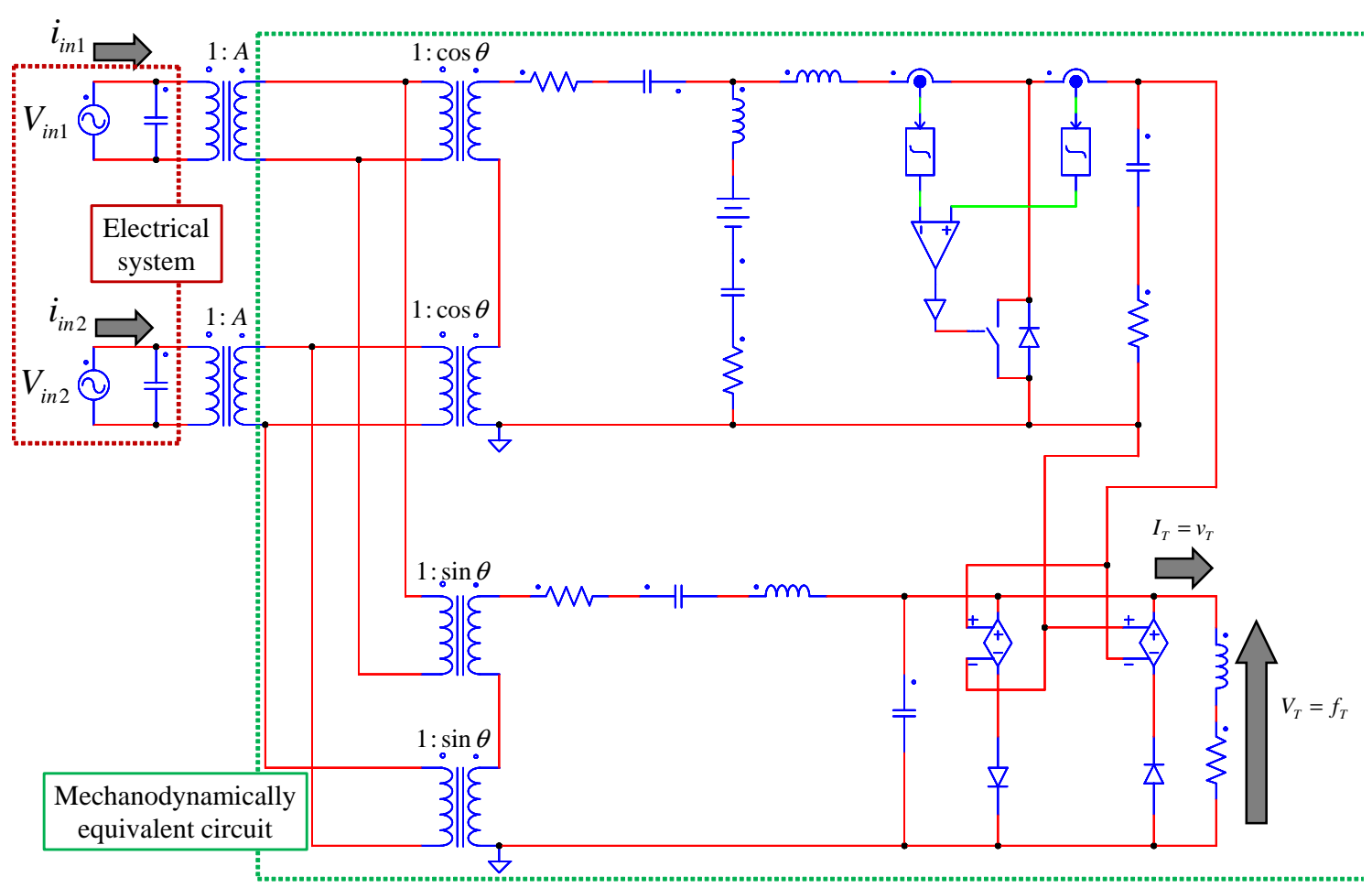

Fig. 9. Electromechanical equivalent circuit of ultrasonic actuator. 


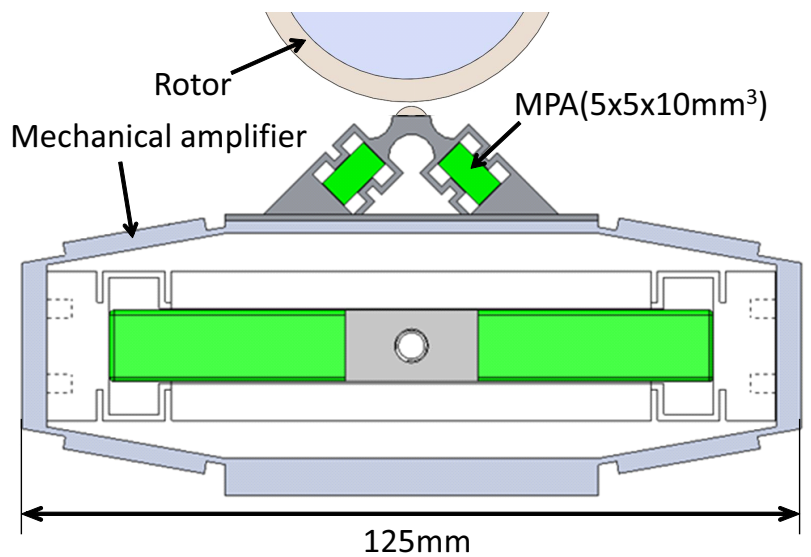

Fig. 10. Construction of ultrasonic actuator. 


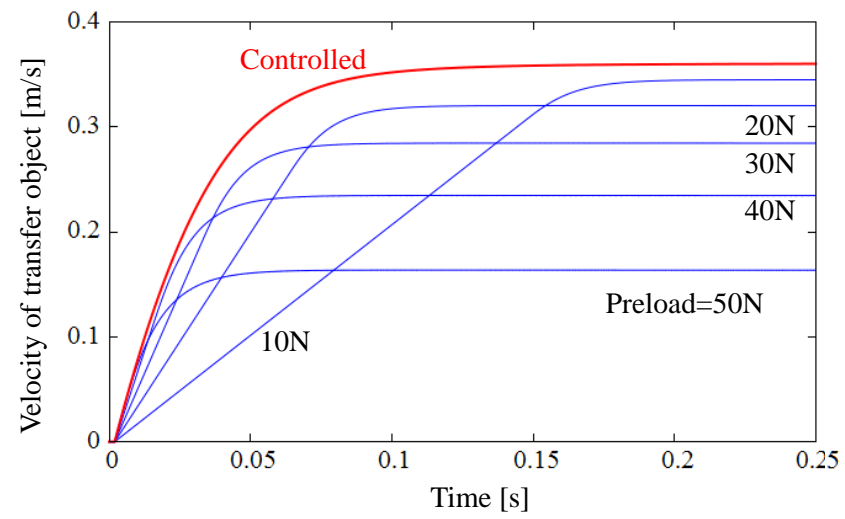

(a)

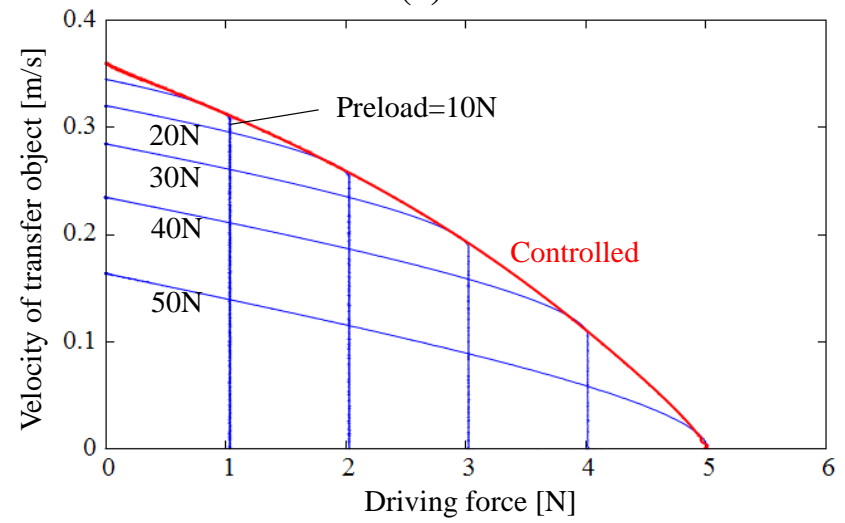

(b)

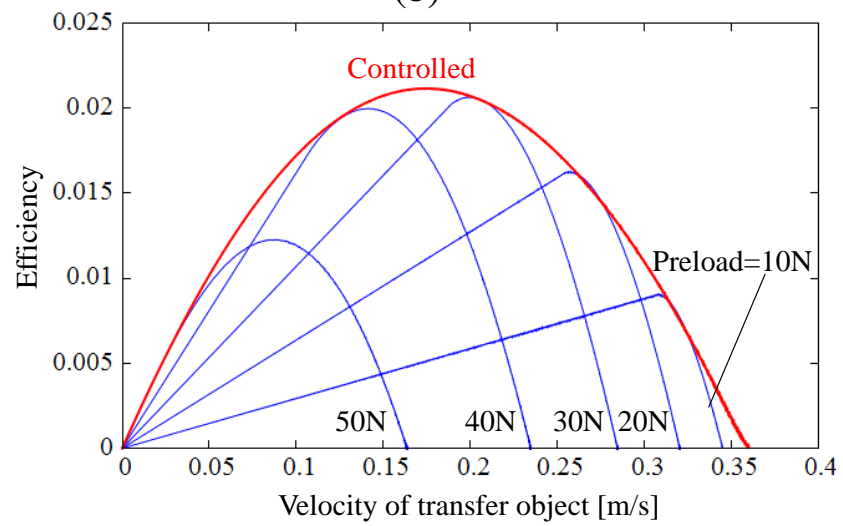

(c)

Fig. 11. Simulation results of electromechanical equivalent circuit with actual values of the tested ultrasonic actuator. (a) Rise time of the velocity of the rotor. (b) Velocity and force characteristics. (c) Efficiency characteristics. 


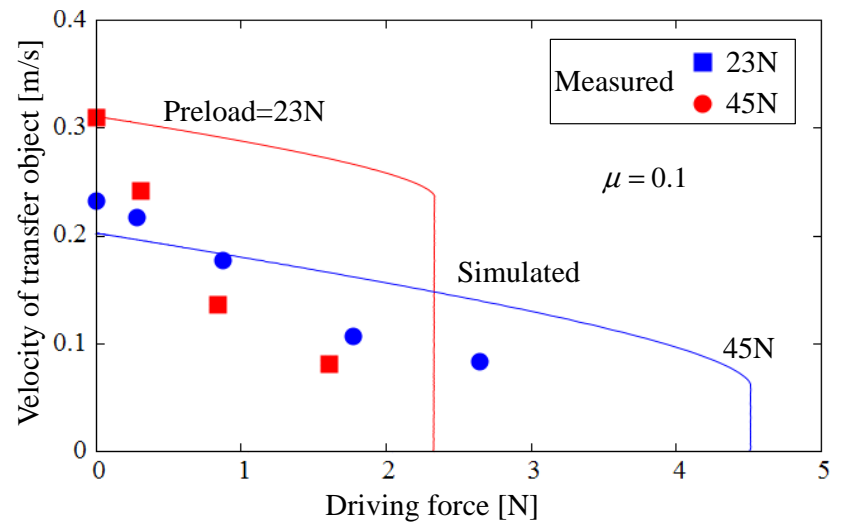

Fig. 12. Comparison of simulated results and experimental results. 


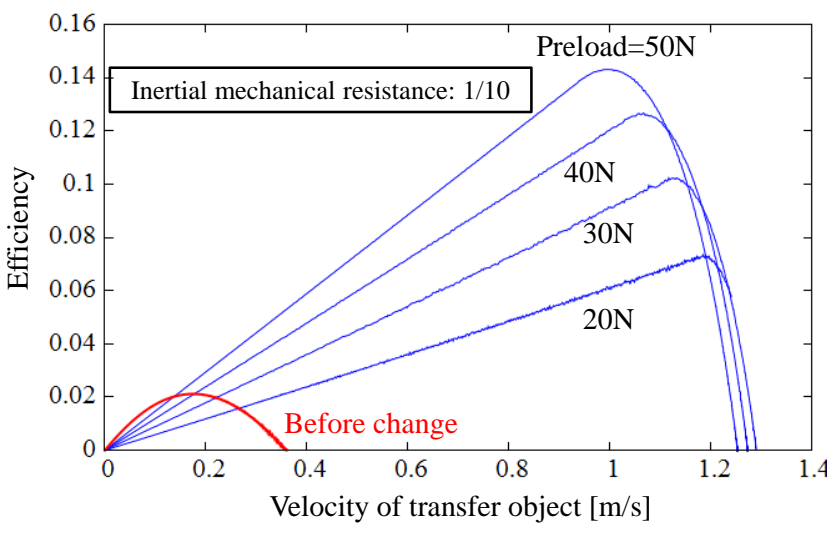

Fig. 13. Efficiency characteristic with internal mechanical resistance reduced to one-tenth. 


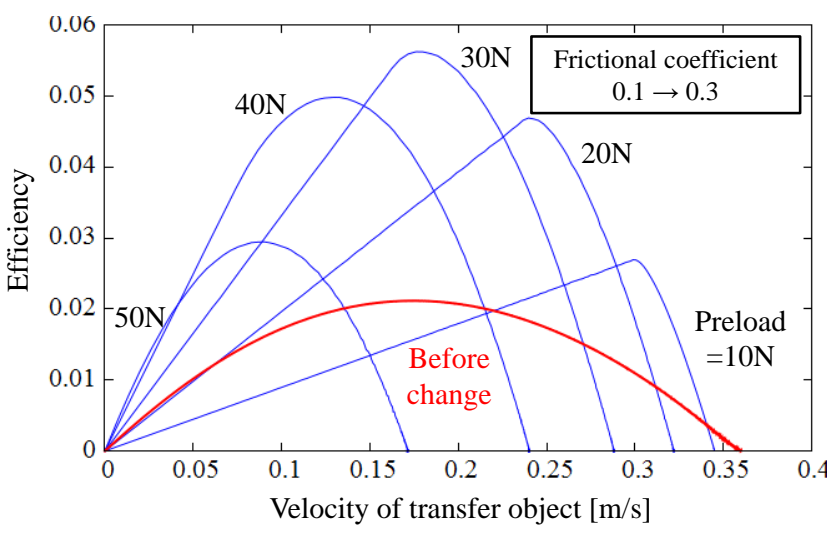

Fig. 14. Efficiency characteristics with increasing frictional coefficient between stator and rotor from 0.1 to 0.3 . 


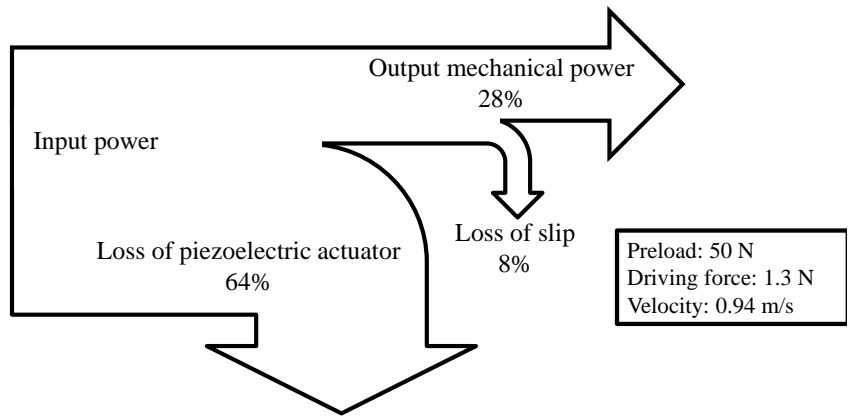

Fig. 15. Example of the power flow of the ultrasonic actuator which employs internal mechanical resistance reduced to one-tenth and frictional coefficient between of the rotor and stator increasing to 0.3 . 